\title{
THE SPINS OF THE FIRST AND SECOND EXCITED STATE OF ${ }^{53} \mathrm{Mn}$
}

\author{
P. H. VUISTER \\ Fysisch Laboratorium der Rijksuniversiteit te Utrecht, Nederland
}

Received 6 October 1966

\begin{abstract}
The ${ }^{52} \mathrm{Cr}(\mathrm{p}, \gamma)^{53} \mathrm{Mn}$ resonances at 1724,1788 and $2149 \mathrm{keV}$ were investigated. Gammagamma angular correlation and gamma polarization measurements are described. The ${ }^{53} \mathrm{Mn}$ levels at 0.38 and $1.29 \mathrm{MeV}$, and the 1788 and $2149 \mathrm{keV}$ resonances have $J^{\pi}=\frac{5}{2}^{(-)}, \frac{3-}{2}{ }^{-}, \frac{1}{2}$ and $\frac{5}{2}$, respectively. The multipole mixing ratio of the $0.38 \mathrm{MeV}$ gamma transition is $x--0.61 \pm 0.08$.

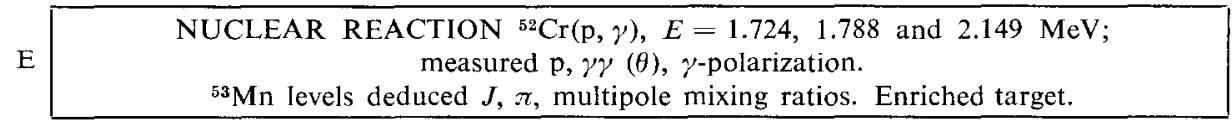

\section{Introduction}

Shell-model calculations predict ${J^{\pi}}^{\pi} \frac{5}{2}^{-}$and $\frac{3}{2}^{-}$for the spins and parities of the first and second excited state of ${ }^{53} \mathrm{Mn}$ at 0.38 and $1.29 \mathrm{MeV}$, respectively ${ }^{1}$ ). The M1 transition strength of the gamma rays de-exciting these levels is expected to be suppressed $^{2}$ ).

The aim of this paper is an experimental verification of these calculations from an investigation of the ${ }^{52} \mathrm{Cr}(\mathrm{p}, \gamma){ }^{53} \mathrm{Mn}$ reaction. Spins, parities and multipole mixing ratios are deduced from $(\mathrm{p}, \gamma \gamma)$ angular correlation and gamma-ray polarization measurements.

Literature data used in the analysis are the $J^{\pi}=\frac{7}{2}^{-}$spin and parity of the ${ }^{53} \mathrm{Mn}$ ground state ${ }^{3}$ ) and the $l_{\mathrm{p}}=1$ stripping pattern ${ }^{4,5}$ ) of the deuteron group to ${ }^{53} \mathrm{Mn}(1.29)$ in the reaction ${ }^{52} \mathrm{Cr}\left({ }^{3} \mathrm{He}, \mathrm{d}\right){ }^{53} \mathrm{Mn}$. The decay schemes of the resonances investigated, were found in a previous experiment ${ }^{6}$ ).

\section{Experiment and method of analysis}

\subsection{ANGULAR CORRELATIONS}

Spins of nuclear levels, and multipole mixing ratios of gamma rays de-exciting these levels can be determined from measured angular distributions and correlations of gamma rays from a proton capture resonance with sharp spin and parity. The expected distributions and correlations, $W\left(J_{1} J_{2} J_{3}, x y, \theta_{1} \theta_{2} \phi\right)$, can be derived from formulae given in refs. ${ }^{7-9}$ ). 
The definitions of the spins $J_{i}$ and of the multipole mixing ratios $x$ and $y$ are given in fig. 1 . The angles between the direction of the incoming protons and the directions of the first and second gamma rays in a cascade are denoted by $\theta_{1}$ and $\theta_{2}$, respectively. The angle between the two planes, defined by the proton beam and the first and second gamma ray, respectively, is given by $\phi$.

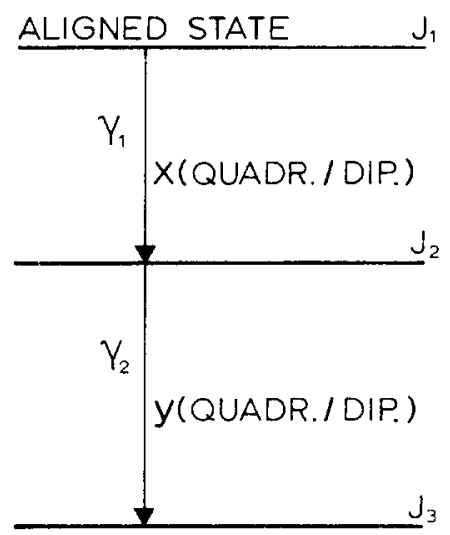

Fig. 1. Definition of quantities used in the angular correlation formalism.

\subsection{GAMMA-RAY POLARIZATION}

The degree of polarization, $P$, of a gamma ray emitted by an aligned state, is a function of the spins and parities of the states concerned, and of the mixing ratio of the gamma ray ${ }^{7,9,10}$ ). The expected absolute value of $P$ can be calculated from $A_{2}$ and $A_{4}$, the coefficients of a Legendre polynomial expansion of the measured angular distribution, and from the possible spin and radiation maxing ratio combinations found from the angular correlation measurements ${ }^{9-11}$ ). A measurement of the sign and absolute value of $P$ then may result in a rejection of spin, parity and mixing ratio combinations that could not be excluded on the basis of angular correlation measurements only.

\subsection{APPARATUS}

The experiments were performed with the proton beam of the Utrecht $3 \mathrm{MV}$ Van de Graaff accelerator. A target of electromagnetically separated ${ }^{52} \mathrm{Cr}, 7 \mu \mathrm{g} / \mathrm{cm}^{2}$ in thickness, on a $0.3 \mathrm{~mm}$ thick tantalum backing was used ${ }^{6}$ ). The detection apparatus and auxiliary equipment has been described previously ${ }^{6,9}$ ). Angular distributions and correlations of cascades through the first excited state were measured with conventional scaler techniques, and correlations of cascades through the second excited state were measured with a multichannel analyser. The two cylindrical $10 \mathrm{~cm} \times 10 \mathrm{~cm}$ $\mathrm{NaI}(\mathrm{Tl})$ crystals were mounted in an iron shield of $5 \mathrm{~cm}$ thickness on a turntable. Both detectors can rotate around the centre point of this table in a horizontal plane, 
and one of them can also rotate in a vertical plane; the proton beam comes in horizontally.

For the angular correlation measurements the turntable was positioned such that its centre point coincided with the beam spot on the target. The distance from the front face of the crystals to the target was $10 \mathrm{~cm}$. In order to absorb the low energy background originating from Coulomb excitation and bremsstrahlung in the tantalum target backing the front face of the crystals was shielded with $5 \mathrm{~mm}$ of copper. The angular correlation measurements were carried out in the four standard geometries I, II, V and VI (refs. $\left.{ }^{9,12}\right)$ ).

The degree of polarization, $P$, of the $0.38 \mathrm{MeV}$ gamma ray was measured with a Compton polarimeter ${ }^{9,10}$ ). The $2.5 \mathrm{~cm} \times 2.5 \mathrm{~cm} \mathrm{NaI}$ scattering crystal was placed vertically below the target spot; it was centered on the vertical turntable axis. The mean scattering angle, $\vartheta$, from this crystal to the two $10 \mathrm{~cm} \times 10 \mathrm{~cm}$ crystals was $84^{\circ}$. This gives rise to maximum polarization efficiency, $p(\vartheta)$, for the $0.38 \mathrm{MeV}$ gamma rays ${ }^{10}$ ). The mean polarization efficiency of the polarimeter, $\overline{p(\vartheta)}$, for the $0.38 \mathrm{MeV}$ gamma ray was calculated to be 0.70 . A third $10 \mathrm{~cm} \times 10 \mathrm{~cm} \mathrm{NaI}$ crystal, detecting the gamma ray de-exciting the resonance state and feeding the $0.38 \mathrm{MeV}$ level was used as a radiation monitor. The electronic arrangement of the polarization experiment has been described previously ${ }^{9,13}$ ). The eccentricity of the experimental arrangement was determined with the $0.511 \mathrm{MeV}$ gamma rays from a ${ }^{22} \mathrm{Na}$ point source.

\subsection{THE ANALYSIS OF THE MEASURED ANGULAR DISTRIBUTIONS AND CORRELA- TIONS}

The analysis was carried out in two steps.

After the necessary corrections for eccentricity, absorption in the target holder, background and accidental coincidences, the measured distributions and each of the measured geometries of the correlations were least-squares fitted to

$$
W\left(\theta_{i}\right) \propto 1+A_{2} P_{2}\left(\cos \theta_{i}\right)+A_{4} P_{4}\left(\cos \theta_{i}\right) .
$$

The $A_{j}$ of the angular distributions of the primary gamma rays of the cascades were compared with the theoretically expected $A_{j}\left(J_{1} J_{2}, x\right)$. In this way some spin combinations $J_{1} J_{2}$ and/or regions of the multipole mixing ratios $x$ could be excluded from further analysis.

The weighted least-squares sum, $\chi^{2}$, provided by the analysis of the angular correlations in terms of Legendre polynomials, was used to determine the experimental errors, which were in some cases larger than the normally accepted statistical ones. If this sum was larger than 1 the errors in the experimental data, $\Delta N_{i y}$, were multiplied by $|\chi|$. In other words, the error assigned is either the internal or the external error, whichever is the largest.

The analysis of the measured angular correlations of each of the investigated cascades consisted of the calculation of $\chi^{2} \equiv \min Q^{2}(x y) \equiv Q^{2}\left(x_{0} y_{0}\right)$, for the remaining 
spin combinations, where

$$
Q^{2}(x y)=\sum_{i g}\left(\frac{N_{i q}-\alpha_{g} W\left(x y, \theta_{1 i g} \theta_{2 i g} \phi_{i g}\right)}{\Delta N_{i g}}\right)^{2} .
$$

In this sum $i$ labels the measured data, $N_{i q}$, and $g$ the geometries. The normalization factors, $\alpha_{g}$, are calculated for each geometry independently. The function $W$ is calculated with coefficients given in ref. $^{8}$ ). The values of $x_{0}$ and $y_{0}$ and their errors $\Delta x_{0}$ and $\Delta y_{0}$ were found by fitting the $Q^{2}(x y)$ surface to a paraboloid in the vicinity of the minima $\left.{ }^{7,14}\right)$. The method used is similar to that given in ref. ${ }^{15}$ ).

The $0.1 \%$ limit, defined such that the probability of finding, a value of $\chi^{2}$ larger than this limit is $0.1 \%$ for a correct solution, was considered as the boundary between acceptable and unacceptable solutions ${ }^{17}$ ).

The values of the solid angle attenuation factors ${ }^{16}$ ) $Q_{2}$ and $Q_{4}$ used for the 0.38 , 0.91 and $1.29 \mathrm{MeV}$ gamma rays are 0.89 and $0.66,0.90$ and 0.69 , and 0.90 and 0.70 , respectively. The attenuation factors for the primary gamma rays are 0.91 and 0.73 . The analysis was carried out for all spin values $J_{(\text {res })} \leqq \frac{7}{2}$ and for $J^{\pi}(0)=\frac{7}{2}^{-}\left(\right.$ref. $\left.^{3}\right)$ ). Only quadrupole/dipole mixing ratios were taken into account.

\section{Results and discussion}

The coefficients of the Legendre polynomial expansion of the measured angular distributions and correlations, given in table 1, summarize the experimental data.

TABLE 1

The coefficients of the Legendre polynomial expansion of the measured angular distributions and correlations, corrected for absorption and eccentricity.

\begin{tabular}{|c|c|c|c|}
\hline \multicolumn{4}{|r|}{ res $\rightarrow 1.29 \rightarrow 0.38$} \\
\hline & $A_{2} \quad A_{4}$ & $A_{2} \quad A_{4}$ & $A_{2} \quad A_{4}$ \\
\hline \multicolumn{4}{|c|}{$E_{\mathrm{p}}=1724 \mathrm{keV}$} \\
\hline A.D. $\gamma_{2}$ & $-0.55 \pm 0.06+0.04 \pm 0.02$ & & \\
\hline \multicolumn{4}{|c|}{$E_{\mathrm{p}}=1788 \mathrm{keV}$} \\
\hline A.D. $\gamma_{1}$ & & $+0.01 \pm 0.02+0.00 \pm 0.02$ & \\
\hline G I & & $+0.03 \pm 0.05+0.01 \pm 0.05$ & $-0.12+0.04-0.09+0.05$ \\
\hline G II & & $+0.02 \pm 0.05+0.02 \pm 0.05$ & $-0.15 \pm 0.04+0.03 \pm 0.04$ \\
\hline G V & & $-0.04 \pm 0.05-0.01 \pm 0.05$ & $+0.02 \pm 0.05+0.06 \pm 0.05$ \\
\hline G VI & & $-0.02 \pm 0.05-0.01 \pm 0.05$ & $-0.06 \pm 0.04+0.00 \pm 0.04$ \\
\hline
\end{tabular}

$E_{\mathrm{p}}=2149 \mathrm{keV}$

$\begin{array}{llllll}\text { A.D. } \gamma_{1} & +0.37 \pm 0.02-0.01 \pm 0.02 & & & & \\ \text { G I } & +0.69 \pm 0.06+0.03 \pm 0.06 & -0.34 \pm 0.04 & -0.01 \pm 0.04 & -0.38 \pm 0.04 & +0.03 \pm 0.04 \\ \text { G II } & -0.41 \pm 0.06+0.05 \pm 0.06 & +0.09 \pm 0.04 & -0.00 \pm 0.04 & -0.27 \pm 0.04 & +0.01 \pm 0.05 \\ \text { G V } & +0.14 \pm 0.05+0.03 \pm 0.05 & -0.35 \pm 0.04 & +0.01 \pm 0.04 & -0.36 \pm 0.04+0.07 \pm 0.04 \\ \text { G VI } & -0.66 \pm 0.05+0.05 \pm 0.05 & +0.02 \pm 0.04 & -0.02 \pm 0.04 & -0.24 \pm 0.05 & -0.01 \pm 0.05\end{array}$




\subsection{THE $2149 \mathrm{keV}$ RESONANCE}

This resonance decays $50 \%$ to the first and $30 \%$ to the second excited state. As a first step in the analysis, the $\chi^{2}$ criterion was applied to the correlations of the cascade through ${ }^{53} \mathrm{Mn}(0.38)$. All possible spin combinations with $J($ res $) \leqq \frac{7}{2}\left(l_{\mathrm{p}} \leqq 3\right)$, $J(0.38) \leqq \frac{11}{2}$, and $J(0)=\frac{7}{2}$ were considered. Only three of these, the combinations $J($ res $)-J(0.38)=\frac{5}{2}-\frac{5}{2}, \frac{7}{2}-\frac{5}{2}$, and $\frac{7}{2}-\frac{9}{2}$, are acceptable.

This result considerably simplifies the analysis of the correlations of the cascades through the second excited state. One has to consider only the combinations of the spins $J($ res $)=\frac{5}{2}$ or $\frac{7}{2}$ (see above), $J(1.29)=\frac{1}{2}$ or $\frac{3}{2}$ (refs. $\left.{ }^{4,5}\right)$ ), $J(0.38)=\frac{5}{2}$ or $\frac{9}{2}$ (see above) and $J(0)=\frac{7}{2}$. The $\chi^{2}$ criterion then indicates $\frac{5}{2}-\frac{3}{2}-\frac{5}{2}$ as the only acceptable spin sequence for $J($ res $)-J(1.29)-J(0.38)$. The $\chi^{2}$ values for these solutions are of the order of the number of free parameters. The $\chi^{2}$ values for the rejected spin combinations are at least twice the $0.1 \%$ limit.

In conclusion, the correlation measurements at the $2149 \mathrm{keV}$ resonance combined with a $l_{\mathrm{p}}=1$ assignment for the $1.29 \mathrm{MeV}$ level from ${ }^{52} \mathrm{Cr}\left({ }^{3} \mathrm{He}, \mathrm{d}\right){ }^{53} \mathrm{Mn}$ experiments, lead to the following assignments:

$$
\begin{aligned}
& J(\text { res })=\frac{5}{2}, J(1.29)=\frac{3}{2}, J(0.38)=\frac{5}{2}, \\
& x(\text { res } \rightarrow 1.29)=-0.01 \pm 0.02, \\
& x(\text { res } \rightarrow 0.38)=+0.01 \pm 0.04, \\
& y(1.29 \rightarrow 0.38)=-0.18 \pm 0.03 \text { or }+30_{-15}^{+270}, \\
& y(0.38 \rightarrow 0 \quad)=-0.61 \pm 0.08 \text { (or }-2.4 \pm 0.4 \text {; rejected in sect. } 3.3) .
\end{aligned}
$$

The parity of the $0.38 \mathrm{MeV}$ level is most likely odd, since the multipole mixing ratio of the $0.38 \rightarrow 0$ transition, $y=-0.61 \pm 0.08$, would be unusually high for a M2/E1 mixture. Combination of $J(1.29)=\frac{3}{2}$, found above, and $l_{\mathrm{p}}=1$ for the $1.29 \mathrm{MeV}$ level found from the ${ }^{52} \mathrm{Cr}\left({ }^{3} \mathrm{He}, \mathrm{d}\right){ }^{53} \mathrm{Mn}$ reaction $\left.{ }^{4,5}\right)$, leads to the conclusion $J^{\pi}(1.29)=\frac{3}{2}^{-}$.

\subsection{THE $1788 \mathrm{keV}$ RESONANCE}

This resonance, which mainly (50\%) decays through ${ }^{53} \mathrm{Mn}(1.29)$ was investigated to remove the remaining ambiguity in $y(1.29 \rightarrow 0.38)$.

The angular correlations (see table 1) were analysed for the four possible combinations of $J($ res $) \leqq \frac{7}{2}, J(1.29)=\frac{3}{2}$, and $J(0.38)=\frac{5}{2}$. An acceptable $\chi^{2}$ value was found for $J($ res $)=\frac{1}{2}$ and $\frac{5}{2}$. Resonance spin $J=\frac{5}{2}$ can be excluded since the corresponding values of the mixing ratio $y(1.29 \rightarrow 0.38)$ deviate from those found above by more than four times the combined errors. Unfortunately, the rather large regions of possible $y(1.29 \rightarrow 0.38)$ values for $J$ (res) $=\frac{1}{2}$ overlap with both values found at the 2149 $\mathrm{keV}$ resonances and thus do not remove the ambiguity. 
The results at this resonance are:

$$
\begin{aligned}
& J(\text { res })=\frac{1}{2}, \\
& x(\text { res } \rightarrow 1.29)=+0.12 \pm 0.10 \text { or }-2.4 \pm 0.8 .
\end{aligned}
$$

\subsection{THE $1724 \mathrm{keV}$ RESONANCE}

The angular distribution of the $0.38 \mathrm{MeV}$ gamma ray is strongly anisotropic (cf. table 1) and therefore this resonance, which decays $75 \%$ to ${ }^{53} \mathrm{Mn}(0.38)$, is suitable for a polarization measurement on this gamma ray. From the measured anisotropy one calculates (see sect. 2.2.), with the known spins of the ground state and first excited state, $P=+0.01 \pm 0.02$ and $+0.11 \pm 0.03$, for $y(0.38 \rightarrow 0)=-0.61 \pm 0.08$ and $-2.4 \pm 0.4$, respectively. The measured polarization is $P=-0.01 \pm 0.02$. The high value of the mixing ratio can be rejected since the difference between the measured and calculated values of $P$ is more than three times the combined error.

\subsection{REMARKS}

The discussion given above was considerably simplified by the $l_{\mathrm{p}}=1$ assignment to the $1.29 \mathrm{MeV}$ level from the $\left({ }^{3} \mathrm{He}\right.$, d) stripping reaction $\left.{ }^{4,5}\right)$. It was also tried to find unique solutions without the use of this assignment. Therefore a more extended analysis of the angular correlation and polarization measurements was carried out. This more involved analysis, including all values of $J(1.29) \leqq \frac{11}{2}$, essentially led to the same results as those found above. One additional solution, however, with $J_{\mathrm{r}}(1788)=\frac{3}{2}, J_{\mathrm{r}}(2149)=\frac{7}{2}, J(1.29)=\frac{5}{2}$ and $J(0.38)=\frac{5}{2}$, could not be rigorously excluded on the basis of the $0.1 \%$ limit. The combined evidence of relatively high $\chi^{2}$-values, an unusually large mixing ratio, and the $l_{\mathrm{p}}=3$ or 4 capture for the 2149 $\mathrm{keV}$ resonance, makes this solution highly improbable.

\section{Conclusions}

The results of the analysis of all measurements is given in fig. 2 . The measured spins and suggested parity are in agreement with shell-model calculations (ref. ${ }^{18}$ ) and the papers quoted therein). They also agree with the spins found for the first and second excited states of the nuclei ${ }_{20}^{43} \mathrm{Ca}_{23},{ }_{20}^{45} \mathrm{Ca}_{25}$ and ${ }_{23}^{51} V_{28}$ (ref. ${ }^{18}$ )). The ground state configuration of these nuclei and that of ${ }_{25}^{53} \mathrm{Mn}_{28}$ consists of three identical particles or holes in the $\mathrm{f}_{\frac{7}{2}}$ shell. The measured values of the $E 2 / \mathrm{M} 1$ radiation mixing ratios, $y(0.38 \rightarrow 0)=-0.61$ and $y(1.29 \rightarrow 0.38)=-0.18$, and the $60: 40$ intensity ratio of the 1.29 and $0.91 \mathrm{MeV}$ gamma rays, indicate that the first and second excited states cannot be described as pure $f_{\frac{7}{2}}^{\mathrm{n}}$ configurations. Between states of such pure configurations $\mathrm{M} 1$ transitions are forbidden. It may be noticed that the multipole mixing ratios, $y(0.38)=-0.61 \pm 0.08$ and $y(0.91)=-0.18 \pm 0.03$, are equal within the experimental errors to those measured for the corresponding transitions in ${ }^{51} \mathrm{~V}$ (ref. $\left.{ }^{19}\right)$ ). 


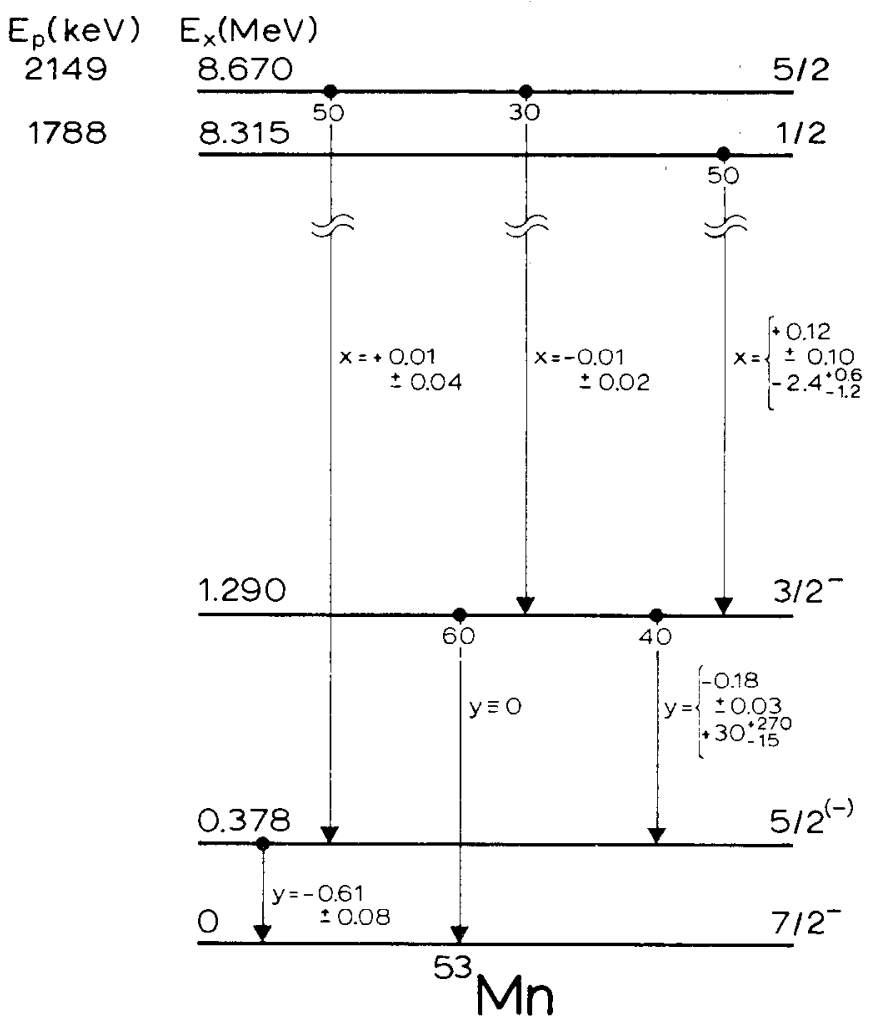

Fig. 2. Final results of the measurements.

The author acknowledges the interest of Professor P. M. Endt in the investigations. Discussions with Dr. D. M. Sheppard and Dr. C. van der Leun led to a better formulation of this paper. The help of H. P. Leenhouts, A. C. Wolff and S. Dijkstra in the experiment is appreciated.

This investigation is partly supported by the joint program of the "Stichting voor Fundamenteel Onderzoek der Materie" and the "Nederlandse Organisatie voor Zuiver Wetenschappelijk Onderzoek".

\section{References}

1) A. de-Shalit, in Selected topics in nuclear theory, ed. by F. Zanouch (IAEA, Vienna, 1963)

2) A. de-Shalit and I. Talmi, Nuclear shell theory (Academic Press, New York, 1963) p. 408

3) W. Dobrowolski, R. V. Jones and C. D. Jeffries, Phys. Rev. 104 (1956) 1378

4) D. D. Armstrong and A. C. Blair, Phys. Rev. 140 (1965) B1226

5) Bachner et al., Jahresbericht 1965, Max-Planck-Institut für Kernphysik, Heidelberg, p. 66

6) P. H. Vuister, Nuclear Physics 83 (1966) 593

7) P. B. Smith, Can. J. Phys. 42 (1964) 1101 
8) P. B. Smith, in Nuclear Reactions II, ed. by P. M. Endt and P. B. Smith (North-Holland Publ. Co., Amsterdam, 1962)

9) F. C. Erné, Nuclear Physics 84 (1966) 241

10) M. Suffert, P. M. Endt and A. M. Hoogenboom, Physica 25 (1959) 659

11) G. Kaye, E. J. C. Read and J. C. Wilmott, Tables of coefficients for the analysis of triple correlations from aligned nuclei, Chadwick Physics Laboratory, University of Liverpool, England, 1963

12) P. W. M. Glaudemans and P. M. Endt, Nuclear Physics 42 (1963) 367

13) N. Hazewindus, Thesis, Delft, Technische Hogeschool, 1964

14) A. Heyligers, Thesis, Utrecht University, 1964

15) P. J. M. Smulders, Physica 31 (1965)

16) P. J. M. Smulders and A. H. E. van Hengel, unpublished, Utrecht, 1965

17) G. J. Nijgh, A. H. Wapstra and R. van Lieshout, Nuclear spectroscopy tables (North-Holland Publ. Co., Amsterdam, 1959) p. 16

18) J. D. McCullen, B. F. Bayman and L. Zamick, Phys. Rev. 134 (1964) B515

19) J. Vervier, Phys. Lett. 5 (1963) 79 